\title{
Erratum to: The significance of hotspots to lekking topi antelopes (Damaliscus lunatus)
}

Jakob Bro-Jørgensen ${ }^{1,2}$

Published online: 9 December 2016

(C) Springer-Verlag Berlin Heidelberg 2016

Erratum to: Behavioral Ecology and Sociobiology 53(5):

324-331

DOI 10.1007/s00265-002-0573-0

In the legend to figure 4 symbols were erroneously confused. The correct legend should read:

Fig. 4 Lek size in relation to density, showing the size of the largest lek within a plain in relation to its topi density. Data from:

- Nyaruhuru and Central plains, ANP, Burrungat plains, MMNR, $\Delta$ Olorukoti plains, MMNR, and $\bullet$ Dutwa plains, SNP (references in Methods)

The online version of the original article can be found at http:// dx.doi.org/10.1007/s00265-002-0573-0.

Jakob Bro-Jørgensen jb24@stir.ac.uk

1 Institute of Zoology, Zoological Society of London, Regent's Park, London NW1 4RY, UK

2 Department of Biological Sciences, University of Stirling, FK9 4LA Stirling, UK 